CERN-TH.7544/94

\title{
Metric Perturbations in Dilaton-Driven Inflation
}

\author{
R. Brustein, M. Gasperini円, M. Giovannini \\ Theory Division, CERN, CH-1211 Geneva 23, Switzerland \\ V. F. Mukhanov \\ Institut fur Theoretische Physik ETH, CH-8093 Zurich, Switzerland \\ and \\ G. Veneziano \\ Theory Division, CERN, CH-1211 Geneva 23, Switzerland
}

\begin{abstract}
We compute the spectrum of scalar and tensor metric perturbations generated, as amplified vacuum fluctuations, during an epoch of dilaton-driven inflation of the type occurring naturally in string cosmology. In the tensor case the computation is straightforward while, in the scalar case, it is made delicate by the appearance of a growing mode in the familiar longitudinal gauge. In spite of this, a reliable perturbative calculation of perturbations far outside the horizon can be performed by resorting either to appropriate gauge invariant variables, or to a new coordinate system in which the growing mode can be "gauged down". The simple outcome of this complicated analysis is that both scalar and tensor perturbations exhibit nearly Planckian spectra, whose common "temperature" is related to some very basic parameters of the string-cosmology background.
\end{abstract}

CERN-TH.7544/94

December 1994

${ }^{1}$ Permanent address: Dipartimento di Fisica Teorica, Via P. Giuria 1, 10125 Turin, Italy. 


\section{Introduction}

According to quantum mechanics, metric and energy-density fluctuations are necessarily present as tiny wrinkles on top of any, otherwise homogeneous, classical cosmological background. It is well known [1] that transitions from one cosmological era to another may lead to a parametric amplification of such perturbations, which eventually reveal themselves as stochastic classical inhomogeneities. In particular, "slow rolling" scenarios leading to de-Sitter like inflation [2] predict an almost scale-invariant spectrum of density (scalar) perturbations [3, 4] and of gravitational waves [5] (for a review, see ref.[6]).

In string cosmology, inflation is expected to be associated with a phase of growing curvature [7] and dilaton coupling [8] (called "pre-big-bang" scenario in [9]), in which the accelerated evolution of the scale factor, $a(t)$, is driven by the kinetic energy of the dilaton field, with negligible contributions from the dilaton potential [9]-15] (see also [16], and [17] for related, though differently motivated, issues in the context of scalar-tensor cosmologies). This inflationary phase is most naturally described in the string frame (S-frame, also called, somewhat improperly, Brans-Dicke frame), in which weakly coupled strings move along geodesic surfaces [18]. In the S-frame, isotropic solutions of the string cosmology equations describe an accelerated expansion of the "pole-inflation" type [19], i.e. one characterized by $\dot{a}>0$, $\ddot{a}>0, \dot{H}>0$, where $H=\dot{a} / a$, and a dot denotes differentiation with respect to cosmic time $t$. In the conformally related Einstein frame (E-frame), in which the curvature term and dilaton kinetic term in the action are diagonalized in the standard canonical form, the corresponding isotropic solutions describe instead an accelerated contraction [10, 11, 13], characterized by $\dot{a}<0, \ddot{a}<0, \dot{H}<0$. In the E-frame the scale factor can be parametrized, in conformal time $\eta(d t \equiv a d \eta)$, as

$$
a \sim(-\eta)^{\alpha}, \quad \alpha>0, \quad \eta \rightarrow 0_{-}
$$

(see [10, 11, 13] for a discussion of how the standard kinematical problems are solved in such a contracting background). We recall, for future reference, that the solution with $\alpha=\frac{1}{2}$ 
corresponds to a pure four-dimensional dilaton-dominated background, while the case $\alpha>\frac{1}{2}$ occurs, in four dimensions, in the presence of additional string matter sources [10, 11].

The epoch of accelerated evolution is assumed to end [7], [8], [9] at some time $|\eta|=\eta_{1}$ when a maximal curvature scale $H_{1} \equiv H\left(\eta_{1}\right)$ is reached, i.e. when higher-derivative terms in the string effective action become important. Both in the S-frame and in the E-frame that point is reached when $a_{1} \eta_{1}=\mathcal{O}\left(\lambda_{s}\right)$, where $\lambda_{s} \simeq \sqrt{\alpha^{\prime} \hbar}$ is the fundamental length of string theory. In the S-frame $\lambda_{s}$ is a constant and the Planck length $\lambda_{p}$ is given by $\lambda_{p}=g_{\text {string }} \lambda_{s}=e^{\frac{1}{2} \varphi} \lambda_{s}$ ( $\varphi$ is the dilaton field), while in the E-frame the fundamental constant is $\lambda_{p}$ (and $\left.\lambda_{s}=e^{-\frac{1}{2} \varphi} \lambda_{p}\right)$. In any case, $H_{1} \lesssim M_{p}\left(M_{p}=G^{-1 / 2}\right)$ if inflation ends when the dilaton is still in the perturbative regime. A smooth transition to standard cosmology at the end of the accelerated pre-big-bang evolution is expected to be controlled both by $\alpha^{\prime}$ corrections to the low energy effective action and by the contribution of a non-perturbative dilaton potential, as discussed in [12] (recent related work concerning the possible smoothing out of curvature singularities in string theory can be found in [20], [21] and [22]).

The accelerated shrinking of the event horizon during inflation [9]-11], [13], and the subsequent transition to a decelerated, radiation-dominated background, produces a dramatic amplification of the initial vacuum fluctuations since, in an inflationary background of the pre-big-bang type, the comoving amplitude of metric perturbations outside the horizon, instead of remaining constant, grows asymptotically [10].

This peculiar effect can be easily illustrated by considering the evolution of tensor metric perturbations in the E-frame, where the background scale factor is given by eq. (1.1), and each Fourier mode of the perturbation satisfies, to lowest order, the simple equation [1]

$$
h_{k}^{\prime \prime}+2 \frac{a^{\prime}}{a} h_{k}^{\prime}+k^{2} h_{k}=0
$$

(a prime denotes differentiation with respect to $\eta$ ). The asymptotic solution of eq. (1.2) well outside the horizon $(|k \eta|<<1)$ is given by

$$
h_{k}=A_{k}+B_{k} \int^{\eta} \frac{d \eta^{\prime}}{a^{2}\left(\eta^{\prime}\right)}=A_{k}+B_{k} \int^{\eta} d \eta^{\prime}\left(-\eta^{\prime}\right)^{-2 \alpha},
$$

where $A_{k}, B_{k}$ are integration constants. For $\alpha<\frac{1}{2}, h_{k}$ approaches a constant asymptotically. The typical amplitude of fluctuations over scales $k^{-1}$, normalized to an initial vacuum- 
fluctuation spectrum, is given as usual by [5, 6]

$$
\left|\delta_{h_{k}}\right|=k^{3 / 2}\left|h_{k}\right| \simeq\left(\frac{H}{M_{p}}\right)_{H C} \simeq \frac{H_{1}}{M_{p}}\left(k \eta_{1}\right)^{1+\alpha}
$$

(the subscript "HC" on a time dependent quantity means that it is to be evaluated at the time of horizon crossing for the particular scale $k^{-1}$ under consideration, i.e. at $|\eta|=\eta_{H C} \simeq k^{-1}$ ).

If, on the contrary, $\alpha \geq \frac{1}{2}$ (which is indeed the case for "realistic" solutions of the string cosmology equations [9, 10, 11]), then the asymptotic solution (1.3) is dominated by the growing mode, and the typical amplitude of tensor perturbations over scales $k^{-1}$ varies in time according to

$$
\left|\delta_{h_{k}}(\eta)\right|=k^{3 / 2}\left|h_{k}\right| \simeq \frac{k \eta}{a^{2} M_{p}}\left(\frac{a}{\eta}\right)_{H C} \simeq\left(\frac{H}{M_{p}}\right)_{H C}\left(\frac{a_{H C}}{a}\right)^{2} k \eta \simeq \frac{H_{1}}{M_{p}}\left(k \eta_{1}\right)^{1+\alpha}|k \eta|^{1-2 \alpha}
$$

with an additional $\ln |k \eta|$ factor appearing at $\alpha=\frac{1}{2}$. As we shall discuss in Sec.2 (see also [10, 11]), the same result is obtained in the conformally related S-frame in which the background metric describes accelerated expansion instead of contraction, and eq. (1.2) is modified by an explicit coupling of the perturbation to the time variation of the dilaton background [23]. We stress that the growth of the comoving amplitude of tensor perturbations can be understood as a consequence of the joint contribution of the metric and of the dilaton background to the "pump" field responsible for the parametric amplification process [24], and is thus to be expected, in general, in case of perturbations evolving in scalar-tensor backgrounds, as noted also in 25.

The final amplitude $\left|\delta_{h_{k}}\left(\eta_{1}\right)\right|$ thus depends on the power $\alpha$ which characterizes the background. In this paper we shall concentrate on the case $\alpha=\frac{1}{2}$ which corresponds to a purely dilaton-driven isotropic inflation in $3+1$ dimensions. From the point of view of string theory, neglecting everything but the dilaton is particularly appealing, since it corresponds to taking a Conformal-Field-Theory as the starting homogeneous background. Furthermore, even if a diluted gas of classical strings is added in the initial conditions, its effect is simply to ignite an accelerated evolution of the flat perturbative vacuum towards the dilaton-driven inflationary regime [11, 13]. The matter contribution becomes eventually negligible and the scale factor ends up evolving (in the E-frame) as $|\eta|^{1 / 2}$. 
The case $\alpha=\frac{1}{2}$ does not pose any problem for tensor perturbations since, according to eq. (1.5), the condition $\left|\delta_{h}\left(\eta_{1}\right)\right| \lesssim 1$ is satisfied for all $\alpha \leq 2$ (provided $H_{1} \lesssim M_{p}$ ), at all scales $k^{-1}>\eta_{1}$ (smaller scales are not parametrically amplified). The situation appears to be drastically different for scalar perturbations, which become instead too large asymptotically to be consistent with the usual description in terms of the linearized gauge-invariant formalism [26, 6]. Consider indeed the canonically normalized field $v_{k}$ associated with scalar perturbations (see Sect. 3). The variable $v / a$ obeys again eq.(1.2), hence behaves asymptotically as in (1.3). Given the relation between $v$ and the scalar metric perturbation $\psi$ in the longitudinal gauge, one finds for the typical amplitude of $\psi$ over scales $k^{-1}>>\eta$ and at time $\eta$ (Section 3):

$$
\left|\delta_{\psi_{k}}(\eta)\right| \simeq \frac{k}{M_{p}|k \eta|^{2}} \frac{\eta}{a^{2}}\left(\frac{a}{\eta}\right)_{\mathrm{HC}} \simeq \frac{H}{M_{p}} \frac{a_{\mathrm{HC}}}{a} \simeq \frac{H}{M_{p}}|k \eta|^{-1 / 2}
$$

For any given $\eta$, there is thus a frequency band defined by the condition $k<\eta^{-1}\left[H / M_{P}\right]^{2}$, for which $\left|\delta_{\psi}\right|>1$, and the perturbative approach apparently breaks down. Alternatively, at sufficiently small $k$, the (naive) spectral energy density evaluated at the end of inflation (i.e. at the beginning of radiation-dominance) is larger than critical,

$$
\Omega_{k}\left(\eta_{1}\right)=\frac{k}{\rho_{c}\left(\eta_{1}\right)} \frac{d \rho}{d k} \simeq\left|\delta_{\psi_{k}}\left(\eta_{1}\right)\right|^{2} \simeq\left(\frac{H_{1}}{M_{p}}\right)^{2}\left(\frac{k_{1}}{k}\right)>1,
$$

$\left(k_{1} \equiv 1 / \eta_{1}\right)$ in contrast with the hypothesis of a negligible back-reaction of the perturbations on the background metric (incidentally, a similar problem was found to arise for scalar perturbations in the context of Kaluza-Klein cosmologies, as a consequence of the shrinking internal dimensions [27, but was left, to the best of our knowledge, unsolved).

Unless the inflationary growth of the background stops at a sufficiently small Hubble parameter $H_{1}$, scalar perturbations apparently do not remain small. In a string theory context, however, the curvature scale at the end of inflation is expected to be the string scale [9]-12], $H_{1} \sim \lambda_{s}^{-1}$. Consequently, a very small value of the string coupling would be required at the end of inflation. Otherwise, a full non-linear approach would seem to be required in order to follow the evolution of scalar perturbations in such a dilaton-dominated background, and to make predictions about their final spectrum. 
The main purpose of this paper is to show that new appropriate perturbative techniques can be developed in order to follow the evolution of such "large" perturbations throughout the inflationary epoch. This will allow us to argue that, because of its special properties, the growing mode does not lead to an inhomogeneous Universe at least if one starts with minimal (i.e. vacuum quantum) primordial fluctuations. In support of this claim we will present explicit first and second order calculations performed in a different - and so far to our knowledge unexploited - "off-diagonal" gauge. The results clearly show that perturbation theory does not break down in the new gauge, that inhomogeneities remain bounded, and that their spectrum can be computed reliably. We shall arrive at similar conclusions by using a different approach based on appropriate covariant and gauge-invariant variables [28, 29]. The spectral amplitude of density fluctuations turns out to coincide, quite unexpectedly, with previous results obtained in the longitudinal gauge [1] by neglecting (with a dubious argument) the growing mode contribution to the scalar perturbation amplitude.

The paper is organized as follows. In Section 2 we recall, for completeness and later comparison, previous works on tensor perturbations in a string cosmology context, and extend it to the case of a dilaton-driven background with extra compactified dimensions. We stress the emergence of tilted spectra, favoring shorter scales, and the stability of the spectrum with respect to the choice of the background solution. In Section 3 we show how the presence of a growing solution for the scalar components of the metric and dilaton perturbations in the longitudinal gauge invalidates a perturbative analysis, typically at small wave numbers and towards the end of the inflationary epoch. We show, in the Appendix, that the growing asymptotic solution can neither be eliminated by an appropriate choice of the number of spatial dimensions, nor by considering anisotropic, Bianchi I type metric backgrounds with an arbitrary number of shrinking internal dimensions. In Section 4, we abandon momentarily the metric perturbation approach in favour of the fluid flow approach pioneered by Hawking [30, extensively applied by Liddle and Lyth [31] and more recently developed by Bruni and Ellis [29]. We show that such variables allow for a consistent direct computation of the spectral energy density of the metric and dilaton fluctuations, without any sign of breakdown of the linear approximation. Computing the size of second order 
corrections directly in these variables looks, however, too difficult a task. Armed with the knowledge that physical observables, such as the energy density stored in the perturbations, remain small, we look, in Section 5, for a more suitable gauge choice incorporating this feature. And, indeed, we are able to identify a new reference frame in which the growing mode at $k=0$ is "gauged away", the small- $k$ growing modes are "gauged down", and a reliable perturbative scheme can be developed. All this is confirmed by an explicit calculation of the relevant second order quadratic terms, which allow us to give an estimate of the size of the second order corrections to metric perturbations. Our main conclusions are finally summarized in Section 6.

\section{Tensor perturbations}

In this Section we recall the main characteristics of tensor perturbations in a dilatondominated background, stressing in particular their stability against the addition of extra dimensions or the choice of different, duality-related solutions of the string cosmology equations.

In the E-frame, the equations obtained from the low energy string effective action [32], for a torsionless background, are simply given by

$$
\begin{gathered}
R_{\mu}{ }^{\nu}-\frac{1}{2} \delta_{\mu}{ }^{\nu} R=\frac{1}{2}\left(\partial_{\mu} \varphi \partial^{\nu} \varphi-\frac{1}{2} \delta_{\mu}{ }^{\nu} \partial_{\alpha} \varphi \partial^{\alpha} \varphi\right) \\
g^{\alpha \beta} \nabla_{\alpha} \nabla_{\beta} \varphi=0
\end{gathered}
$$

where $\varphi$ is the dilaton field and, unless otherwise stated, we shall adopt units in which $16 \pi G=1$. It is well known that eq.(2.2) is a consequence of eq.(2.1), to which we shall therefore restrict our attention from now on. Note that we neglect the contribution of a possible dilaton self-interaction potential having in mind that the whole evolution starts out in the weak coupling region. Looking for spatially flat solutions in which there are $d$ spatial dimensions which evolve in time with a scale factor $a(\eta)$, while other $n$ internal dimensions simultaneously shrink with a scale factor $b(\eta)$,

$$
g_{\mu \nu}=\operatorname{diag}\left(a^{2},-a^{2} \delta_{i j},-b^{2} \delta_{m n}\right), \quad \varphi=\varphi(\eta)
$$




$$
i, j=1, \cdots, d \quad m, n=d+1, \cdots, d+n
$$

the Einstein equations (2.1), (2.2) take the explicit form

$$
\begin{aligned}
d(d-1) \mathcal{H}^{2}+n(n-1) \mathcal{F}^{2}+2 n d \mathcal{H} \mathcal{F} & =\frac{1}{2} \varphi^{\prime 2} \\
2(d-1) \mathcal{H}^{\prime}+(d-1)(d-2) \mathcal{H}^{2}+2 n \mathcal{F}^{\prime}+n(n+1) \mathcal{F}^{2}+2 n(d-2) \mathcal{H} \mathcal{F} & =-\frac{1}{2} \varphi^{\prime 2} \\
2(n-1) \mathcal{F}^{\prime}+2 d \mathcal{H}^{\prime}+d(d-1) \mathcal{H}^{2}+n(n-1) \mathcal{F}^{2}+2(d-1)(n-1) \mathcal{H} \mathcal{F} & =-\frac{1}{2} \varphi^{\prime 2} \\
\varphi^{\prime \prime}+[(d-1) \mathcal{H}+n \mathcal{F}] \varphi^{\prime} & =0
\end{aligned}
$$

where $\mathcal{H}=a^{\prime} / a=a H$ and $\mathcal{F}=b^{\prime} / b$. We shall consider, in particular, the exact anisotropic solution parametrized, for $\eta \rightarrow 0_{-}$, by

$$
\begin{array}{lc}
a=(-\eta)^{\alpha}, \quad b=(-\eta)^{\beta}, & \varphi=\frac{n-d-\sqrt{d+n}}{1+\sqrt{d+n}} \ln (-\eta)+\text { const } \\
\alpha=\frac{\sqrt{d+n}+1-2 n}{(1+\sqrt{d+n})(d+n-1)}, & \beta=\frac{\sqrt{d+n}-1+2 d}{(1+\sqrt{d+n})(d+n-1)}
\end{array}
$$

Such a background is a particularly significant candidate for describing a phase of inflation plus dynamical dimensional reduction in a string cosmology context. Indeed, if one goes over to the S-frame by the conformal transformation:

$$
\widetilde{g}_{\mu \nu}^{\text {string }}=g_{\mu \nu} e^{\frac{2 \varphi}{d+n-1}}
$$

one finds a particular case of the general exact dilaton-driven solution in critical dimensions [8, 11, 33, 34, in which "external" and "internal" scale factors are related by the duality transformation $\tilde{b}=1 / \tilde{a}$.

Each Fourier mode $h_{k}$ of the transverse-traceless tensor perturbations $\delta g_{i j}=-a^{2} h_{i j}(\eta, \vec{x})$ of the "external" $d$-dimensional metric background satisfies, in the E-frame, the free scalar field equation [1, 23, 35]

$$
h_{k}^{\prime \prime}+[(d-1) \mathcal{H}+n \mathcal{F}] h_{k}^{\prime}+k^{2} h_{k}=0
$$

For the solution (2.5), the coefficient of the $h_{k}^{\prime}$ term of this equation is exactly dimensionalityindependent, since

$$
(d-1) \frac{a^{\prime}}{a}+n \frac{b^{\prime}}{b}=[(d-1) \alpha+n \beta] \frac{1}{\eta}=\frac{1}{\eta}
$$


It follows that, in the long wavelength $(|k \eta| \rightarrow 0)$ limit,

$$
h_{k}=A_{k}+B_{k} \ln |k \eta| .
$$

Tensor perturbations are thus growing logarithmically in a dilaton-driven inflationary background, quite irrespectively of the isotropy and of the number of spatial dimensions. This mild growth, however, does not prevent a linearized metric perturbation description of the vacuum fluctuations, in any number of dimensions. Consider, in fact, the correctly normalized variable $u_{k}$ satisfying canonical commutation relations, which for tensor perturbations in the background (2.3) is related to $h_{k}$ by

$$
u_{k}=y h_{k}, \quad y=a^{(d-1) / 2} b^{n / 2} .
$$

$u_{k}$ satisfies the equation [23, 10]

$$
u_{k}^{\prime \prime}+\left(k^{2}-\frac{y^{\prime \prime}}{y}\right) u_{k}=0
$$

with asymptotic solution, for $|k \eta|<<1$,

$$
u_{k}=c_{1} y+c_{2} y \int^{\eta} \frac{d \eta^{\prime}}{y^{2}\left(\eta^{\prime}\right)}
$$

( $c_{1}$ and $c_{2}$ are integration constants). Inside the horizon $(|k \eta|>>1)$, the amplitude of a freely oscillating, positive frequency mode, normalized to the initial vacuum state at $\eta=-\infty$, is represented by $\left|u_{k}\right| \simeq k^{-1 / 2}$. Since $y^{2} \sim|\eta|$, we thus obtain for the normalized vacuum fluctuations outside of the horizon

$$
h_{k}=\frac{u_{k}}{y} \simeq \frac{\ln |k \eta|}{\sqrt{k} y_{\mathrm{HC}}},
$$

which gives a typical amplitude over scales $k^{-1}$

$$
\left|\delta_{h_{k}}(\eta)\right| \simeq\left(\frac{H_{1}}{M_{p}}\right)^{(d+n-1) / 2}\left(k \eta_{1}\right)^{(d+n) / 2} \ln |k \eta|
$$

(we have assumed a final inflation scale $H_{1}$ of the same order as the final compactification scale, $\left.H_{1} \simeq\left(a_{1} \eta_{1}\right)^{-1} \simeq\left(b_{1} \eta_{1}\right)^{-1}\right)$. The necessary condition for the validity of the linear 
approximation, $\left|\delta_{h}\right|<1$, is therefore satisfied for any $d$, and for all scales $k<k_{1}=1 / \eta_{1}$, provided that $H_{1} \lesssim M_{P}$, i.e. that the dilaton $\varphi$ is still in the perturbative region $\left(e^{\varphi} \lesssim 1\right)$, at the end of inflation. For future reference, we explicitly write the result for the $n=0, d=3$ case, corresponding to an isotropic four-dimensional Universe,

$$
\left|\delta_{h_{k}}(\eta)\right| \simeq\left(\frac{H_{1}}{M_{p}}\right)\left(k \eta_{1}\right)^{3 / 2} \ln |k \eta| .
$$

We note, finally, that the same results are obtained if tensor perturbations are linearized in the S-frame, related to the E-frame by the conformal transformation (2.6). Indeed, in the S-frame, the dilaton contribution appears explicitly in the tensor perturbation equation, which becomes 23.

$$
h_{k}^{\prime \prime}+\left[(d-1) \widetilde{\mathcal{H}}+n \widetilde{\mathcal{F}}-\varphi^{\prime}\right] h_{k}^{\prime}+k^{2} h_{k}=0
$$

where $\tilde{\mathcal{H}}=\tilde{a}^{\prime} / \tilde{a}, \tilde{\mathcal{F}}=\tilde{b}^{\prime} / \tilde{b}=-\tilde{\mathcal{H}}$ (conformal time is the same in both frames). The conformal transformation (2.6) leads to

$$
\widetilde{a}=\widetilde{b}^{-1}=(-\eta)^{-1 /(\sqrt{d+n}+1)}
$$

so that we obtain

$$
h_{k}^{\prime \prime}+\frac{1}{\eta} h_{k}^{\prime}+k^{2} h_{k}=0
$$

which implies, asymptotically, the same logarithmic mild growth (2.9), as before. This is in complete agreement with the frame-independence of the perturbation spectrum, already stressed in [10, 11].

\section{The growing mode of scalar perturbations}

We now turn to scalar perturbations, considering a four-dimensional, conformally flat cosmological background

$$
g_{\mu \nu}=a^{2} \operatorname{diag}\left(1,-\delta_{i j}\right), \quad \varphi=\varphi(\eta)
$$

for which the Einstein equations can be written as

$$
12 \mathcal{H}^{2}=\varphi^{\prime 2}
$$




$$
\begin{aligned}
8 \mathcal{H}^{\prime}+4 \mathcal{H}^{2} & =-\varphi^{\prime 2} \\
\varphi^{\prime \prime}+2 \mathcal{H} \varphi^{\prime} & =0
\end{aligned}
$$

and the general expression for the (scalar part) of the perturbed line element has the well known form (see for example [6])

$$
d s^{2}=a^{2}(1+2 \phi) d \eta^{2}-a^{2}\left[(1-2 \psi) \delta_{i j}+2 \partial_{i} \partial_{j} E\right] d x^{i} d x^{j}-2 a^{2} \partial_{i} B d x^{i} d \eta
$$

A popular choice for discussing scalar perturbations of the metric and of the matter sources (in this case the dilaton field) is the so-called longitudinal gauge $(E=0=B)$, where

$$
\begin{array}{cc}
\delta g_{00}=2 a^{2} \phi, \quad \delta g_{i j}=2 a^{2} \psi \delta_{i j}, \quad \delta g^{00}=-\frac{2}{a^{2}} \phi, \quad \delta g^{i j}=-\frac{2}{a^{2}} \psi \delta^{i j} \\
\delta g_{0 i}=0=\delta g^{0 i}, \quad \delta \varphi=\chi
\end{array}
$$

and where $\phi$ and $\psi$ turn out to coincide (to first order) with the two gauge invariant Bardeen variables [26, 6]. By perturbing Einstein's equations in this gauge, we get, from the offdiagonal spatial components, the condition $\phi=\psi$. The remaining perturbation equations when written explicitly in terms of $\psi$ and $\chi$, take the form [11, 6]

$$
\begin{gathered}
\nabla^{2} \psi-3 \mathcal{H} \psi^{\prime}=\frac{1}{4} \varphi^{\prime} \chi^{\prime} \\
\psi^{\prime \prime}+3 \mathcal{H} \psi^{\prime}=\frac{1}{4} \varphi^{\prime} \chi^{\prime} \\
\chi^{\prime \prime}+2 \mathcal{H} \chi^{\prime}-\nabla^{2} \chi=4 \varphi^{\prime} \psi^{\prime}
\end{gathered}
$$

with the additional constraint

$$
\psi^{\prime}+\mathcal{H} \psi=\frac{1}{4} \varphi^{\prime} \chi
$$

Their combination gives the decoupled equation for the Fourier mode $\psi_{k}\left(\nabla^{2} \psi_{k}=-k^{2} \psi_{k}\right)$

$$
\psi_{k}^{\prime \prime}+6 \mathcal{H} \psi_{k}^{\prime}+k^{2} \psi_{k}=0
$$

The solution of equations (3.2) representing, in the S-frame, a dilaton-driven, accelerated inflationary background corresponds, in the E-frame, to a growing dilaton field, and to an 
accelerated contraction. The asymptotic behaviour of such a background for $\eta \rightarrow 0_{-}$can be parametrized in conformal time as [11]

$$
a(\eta)=(-\eta)^{1 / 2}, \quad \varphi(\eta)=-\sqrt{12} \ln a .
$$

Eq.(3.9) is solved asymptotically $(|k \eta|<<1)$ by

$$
\psi_{k}=c_{1}(k) \ln |k \eta|+c_{2}(k) \frac{1}{\eta^{2}}
$$

showing that the mode $\psi_{k}$, far from being frozen outside the horizon, grows in time like $\eta^{-2}$.

If we are interested, in particular, in the evolution of primordial vacuum fluctuations, the correct normalization of $\psi_{k}$ is to be fixed in terms of the variable $v$ satisfying canonical commutation relations. One has [6]:

$$
\begin{aligned}
\psi_{k} & =-\frac{\varphi^{\prime}}{4 M_{P} k^{2}}\left(\frac{v_{k}}{a}\right)^{\prime} \\
v & =a\left(\chi+\frac{\varphi^{\prime}}{\mathcal{H}} \psi\right)
\end{aligned}
$$

where $v$, which has correct canonical dimensions $\left[v_{k}\right]=[k]^{-1 / 2}$, satisfies the equation

$$
v_{k}^{\prime \prime}+\left(k^{2}-\frac{a^{\prime \prime}}{a}\right) v_{k}=0 .
$$

Note that this equation is precisely the same as eq.(2.11) for tensor perturbations. In the background (3.10) the exact solution of this equation, which represents for $|k \eta|>>1$ a freely oscillating positive frequency mode normalized to the vacuum state at $\eta \rightarrow-\infty$, is given in terms of the second-type Hankel function $H_{\nu}^{(2)}$ as

$$
v_{k}(\eta)=\eta^{1 / 2} H_{0}^{(2)}(|k \eta|) \underset{\eta \rightarrow-\infty}{\longrightarrow} \frac{1}{\sqrt{k}} e^{-i k \eta}
$$

Far outside the horizon, $|k \eta|<<1$, one obtains the asymptotic normalized expression

$$
\left|v_{k}(\eta)\right| \simeq \frac{a}{a_{\mathrm{HC}}} \frac{|\ln (-k \eta)|}{\sqrt{k}}
$$

which, when inserted into eq. (3.12), yields the following expression for the typical amplitude of fluctuations on length scales $k^{-1}$ at time $\eta$,

$$
\begin{aligned}
\left|\delta_{\psi_{k}}(\eta)\right| & =k^{3 / 2}\left|\psi_{k}(\eta)\right| \simeq \frac{1}{M_{p}|k \eta|^{2}}\left(\frac{k}{a}\right)_{\mathrm{HC}} \\
& \simeq \frac{H_{1}}{M_{p}} \frac{\left|k \eta_{1}\right|^{3 / 2}}{|k \eta|^{2}} .
\end{aligned}
$$


(to obtain the last equality we have multiplied and divided by the final inflationary scale $\left.H_{1} \simeq\left(a_{1} \eta_{1}\right)^{-1}\right)$. In our background (3.10), the linear approximation (i.e. $\left|\delta \psi_{k}\right|<1$ ) is thus only valid on scales $k$ such that

$$
\left|\frac{\eta}{\eta_{1}}\right| \gtrsim\left(\frac{H_{1}}{M_{p}}\right)^{1 / 2}\left(\frac{k_{1}}{k}\right)^{1 / 4} .
$$

As an example, for a nearly Planckian inflation scale $H_{1}$, this condition implies that fluctuations over the scale presently probed by COBE observations can be treated perturbatively only for $|\eta|>10^{7} \eta_{1}\left(|t|>10^{10} t_{p}\right.$, in cosmic time). For a similar result in a Kaluza-Klein context see ref. [27].

It is amusing to observe that this conclusion can be evaded in the case of a background with $d>3$ isotropic spatial dimensions, in spite of the fact that the solution is still growing in time. We refer to the Appendix for a detailed discussion of this case, as well as for a discussion of scalar perturbations in the higher-dimensional anisotropic background (2.5). The way out of this apparently disastrous result will be discussed in the next two Sections.

\section{Covariant approach to scalar perturbations}

As discussed in the previous Section, the growth of scalar metric perturbations outside the horizon in the longitudinal gauge seems to imply that, in general, a linearized approach is not sufficient for a complete and consistent description of the evolution of scalar metric and dilaton perturbations in a dilaton-driven inflationary background. This is somewhat surprising, since on general grounds one would expect physical observables over a given scale to freeze out when such a scale goes outside the horizon. In this Section, by using the fully covariant and gauge-invariant formalism recently proposed in [29], we will show that the total energy density contained in the perturbations is small compared to that of the background so that the physical situation is well described by a nearly homogeneous background.

Within the covariant approach, one has to define two appropriate variables, $\Delta$ and $C$, characterizing the evolution of density and curvature inhomogeneities. For a scalar field dominated background, such variables are related, respectively, to the comoving spatial 
Laplacian of the momentum density $\left|\nabla_{\mu} \varphi\right|$ of the scalar field, and to the comoving spatial Laplacian of the spatial part of the scalar curvature. Here "spatial" means orthogonal to the direction of the four vector $\nabla_{\mu} \varphi$, assumed to be time-like, and defining the preferred world-lines of comoving observers. The exact definition of these variables for $d=3$, and in the absence of scalar field potential is [29]

$$
\begin{gathered}
\Delta=2 a h_{\mu}^{\alpha} \nabla_{\alpha}\left(\frac{a}{f} h^{\mu \beta} \nabla_{\beta} f\right) \\
C=a h_{\mu}^{\alpha} \nabla_{\alpha}\left(a^{3} h^{\mu \beta} \nabla_{\beta}{ }^{(3)} R\right)
\end{gathered}
$$

where $f$ is the momentum density magnitude

$$
f=\sqrt{\nabla_{\mu} \varphi \nabla^{\mu} \varphi}
$$

$h_{\mu \nu}$ is the projection tensor on the 3 -space orthogonal to the momentum,

$$
h_{\mu \nu}=g_{\mu \nu}-u_{\mu} u_{\nu}, \quad u_{\mu}=-\frac{1}{f} \nabla_{\mu} \varphi,
$$

and ${ }^{(3)} R$ is the Ricci scalar of the spatial sub-manifold orthogonal to $u^{\mu}$, defined in terms of the local expansion parameter $\Theta$, and the shear tensor $\sigma_{\mu \nu}$, as

$$
\begin{aligned}
{ }^{(3)} R & =-\frac{2}{3} \Theta^{2}+\sigma_{\mu \nu} \sigma^{\mu \nu}+\frac{1}{2} \nabla_{\mu} \varphi \nabla^{\mu} \varphi \\
\Theta & =-\nabla_{\mu}\left(\frac{1}{f} \nabla^{\mu} \varphi\right) \\
\sigma_{\mu \nu} & =-\frac{1}{f} h_{\mu}{ }^{\alpha} h_{\nu}{ }^{\beta} \nabla_{\alpha} \nabla_{\beta} \varphi-\frac{1}{3} h_{\mu \nu} \Theta .
\end{aligned}
$$

The variables $\Delta$ and $C$ satisfy exact equations which are obtained by taking the spatial gradient of the energy conservation and of the Raychaudury equation [28].

From now on we will assume that the unperturbed background is a three-dimensional, spatially flat isotropic manifold described by the solution (3.10) of the string cosmology equations, and we shall perform a perturbative expansion around this background, in terms of the variables $\Delta$ and $C$. One finds that, to zeroth order, the background values of $\Delta$ and $C$ are both vanishing, an obvious result for variables representing density and curvature 
fluctuations when computed in a perfectly homogeneous manifold. This is, in fact, the main reason why these variables are particularly suited for our situation.

To the first order, by linearizing around the given background the exact equations satisfied by $\Delta$ and $C$, we find the following set of coupled first order equations

$$
\begin{aligned}
2 \mathcal{H} \Delta^{\prime} & =C \\
C^{\prime} & =2 \mathcal{H} \nabla^{2} \Delta
\end{aligned}
$$

which yields, upon differentiation, a set of decoupled second order equations for the Fourier modes $\Delta_{k}, C_{k}$

$$
\begin{aligned}
\Delta_{k}^{\prime \prime}-2 \mathcal{H} \Delta_{k}^{\prime}+k^{2} \Delta_{k} & =0 \\
C_{k}^{\prime \prime}+2 \mathcal{H} C_{k}^{\prime}+k^{2} C_{k} & =0
\end{aligned}
$$

The general exact solution of these equations can be written in terms of Hankel functions of the first and second kind

$$
\begin{aligned}
\Delta_{k} & =c_{1} \eta H_{1}^{(1)}(k \eta)+c_{2} \eta H_{1}^{(2)}(k \eta) \\
C_{k} & =c_{3} H_{0}^{(1)}(k \eta)+c_{4} H_{0}^{(2)}(k \eta) .
\end{aligned}
$$

The above solution is consistent with the system (4.8) provided

$$
c_{3}=k c_{1}, \quad c_{4}=k c_{2} .
$$

From (4.10) we obtain the following asymptotic $(|k \eta| \rightarrow 0)$ behaviour for $\Delta$ and $C$

$$
\begin{aligned}
\Delta_{k} & =A_{1}(k)+\left[A_{2}(k)+A_{3}(k) \ln |k \eta|\right]|k \eta|^{2} \\
C_{k} & =B_{1}(k)+B_{2}(k) \ln |k \eta| .
\end{aligned}
$$

In this expression, only two of the coefficients $A_{1,2,3}, B_{1,2}$ are arbitrary integration constants, while the others follow from the condition (4.11) and the small argument limit of the Hankel functions.

The fact that, in the linear approximation, $\Delta_{k}$ and $C_{k}$ stay constant outside the horizon, with at most the logarithmic variation already found for tensor perturbations (see Section 
2), suggests that such variables could provide a consistent linearized description of the evolution of vacuum fluctuations in terms of a perturbative expansion around a homogeneous background. To check this, we first have to normalize $\Delta_{k}$ and $C_{k}$ to the vacuum fluctuation spectrum, by relating them to the canonical variable $v$ which defines the initial vacuum state at $\eta \rightarrow-\infty$. This can always be done, for any given mode $k$, by expressing $\Delta_{k}$ and $C_{k}$ to first order in terms of the metric and dilaton scalar perturbation variables, at early enough time scales, when the linear approximation is valid also in the longitudinal gauge. Such a relation between linearized variables can be consistently established even for modes outside of the horizon, as discussed in the previous Section, provided the corresponding time scale $\eta$ is in the interval (see eq. (3.17))

$$
\left(\frac{k_{1}}{k}\right)^{1 / 4}<\left|\frac{\eta}{\eta_{1}}\right|<\frac{k_{1}}{k}
$$

(we have assumed $H_{1} \lesssim M_{P}$ ).

By computing $\delta f, \delta h_{\mu \nu}$, and $\delta^{(3)} R$ to first order in the scalar perturbations (3.4), and using the background equations (3.2), we obtain from the exact definitions of $\Delta$ and $C$ (4.1), (4.2) their explicit relation to the metric perturbation variables, valid in the linear approximation,

$$
\begin{gathered}
\Delta=2 \nabla^{2}\left(\frac{3 \mathcal{H}}{\varphi^{\prime}} \chi-\psi+\frac{\chi^{\prime}}{\varphi^{\prime}}\right) \\
C=\frac{4 \mathcal{H}}{\varphi^{\prime}} \nabla^{2}\left(\nabla^{2} \chi+3 \varphi^{\prime} \psi^{\prime}+3 \mathcal{H} \chi^{\prime}\right)
\end{gathered}
$$

These two relations have the remarkable feature that, while each term on the right hand side grows, asymptotically, as $1 / \eta^{2}$ or $1 / \eta^{4}$, the particular combinations entering in $\Delta$ and $C$ lead to an exact cancellation of the growing mode contribution and reproduce the "regularized" asymptotic behaviour (4.12). This cancellation can be explicitly displayed by noticing that, using the background equations, the perturbation equations (3.5)-(3.8), and the definitions (3.12), the terms on the right hand side of eqs. (4.14), (4.15) can be combined to give

$$
\begin{aligned}
\Delta & =\frac{2}{\varphi^{\prime}} \nabla^{2}\left(\frac{v}{a}\right)^{\prime} \\
C & =\frac{4 \mathcal{H}}{\varphi^{\prime}} \nabla^{2} \nabla^{2}\left(\frac{v}{a}\right)
\end{aligned}
$$


By inserting now the asymptotic solution (3.15) for the mode $v_{k}$, we obtain for $\Delta_{k}$ and $C_{k}$ the normalized asymptotic behaviour

$$
\begin{aligned}
\left|\Delta_{k}\right| & \simeq \frac{\sqrt{k}}{M_{p}|a \eta|_{\mathrm{HC}}} \\
\left|C_{k}\right| & \simeq \frac{k^{5 / 2}}{M_{p}|a \eta|_{\mathrm{HC}}} \ln |k \eta|
\end{aligned}
$$

in full agreement with eq. (4.12).

Once we have the normalized behaviour of $\Delta_{k}$ and $C_{k}$ we can check the validity of the linear approximation for such variables. The typical amplitudes of the vacuum fluctuations described by $\Delta$ and $C$ over length scales $k^{-1}$ can be estimated, respectively, as $k^{3 / 2}\left|\Delta_{k}\right|$ and $k^{3 / 2}\left|C_{k}\right|$. An approximate description of $\Delta$ and $C$ as small perturbations around a homogeneous background is consistent provided their amplitude is smaller than the magnitude of the corresponding terms obtained by replacing spatial with temporal gradients in the exact definitions (4.1), (4.2). Such terms are typically of order $\eta^{-2}$ for $\Delta$ and $\eta^{-4}$ for $C$. A linearized description of the evolution of the vacuum fluctuations in terms of $\Delta$ and $C$ is thus consistent if

$$
\begin{aligned}
\frac{|k \eta|^{2}}{M_{p}|a \eta|_{\mathrm{HC}}} & \simeq \frac{H_{1}}{M_{p}}|k \eta|^{2}\left|k \eta_{1}\right|^{3 / 2} \ln |k \eta|<1 \\
\frac{|k \eta|^{4}}{M_{p}|a \eta|_{\mathrm{HC}}} \ln |k \eta| & \simeq \frac{H_{1}}{M_{p}}|k \eta|^{4}\left|k \eta_{1}\right|^{3 / 2} \ln |k \eta|<1
\end{aligned}
$$

Both conditions are clearly satisfied, at all $|\eta| \geq \eta_{1}$, for all $k \leq 1 / \eta$, provided $H_{1} \lesssim M_{P}$.

As a consistency check we can easily verify that the spectral amplitude of the fluctuations $\delta \rho / \rho$ of the comoving source energy density, defined in the linear approximation as

$$
k^{3 / 2}\left|\frac{\delta \rho_{k}}{\rho}\right| \simeq \frac{1}{\sqrt{k}}\left|\Delta_{k}\right| \simeq \frac{H_{1}}{M_{p}}\left|k \eta_{1}\right|^{3 / 2} \ln |k \eta|
$$

is smaller than critical for any mode $k \leq k_{1}=\eta_{1}^{-1}$. This justifies the fact that the evolution of the vacuum fluctuations is treated linearly, neglecting their back-reaction on the original geometry.

Note that the previous equation defines fluctuations of the total comoving energy density, where comoving is referred to the time-like momentum of the scalar field, i.e.

$$
\rho=T_{\mu \nu} u^{\mu} u^{\nu}=\left(\partial_{\mu} \varphi \partial_{\nu} \varphi-\frac{1}{2} g_{\mu \nu} \partial_{\alpha} \varphi \partial^{\alpha} \varphi\right) \frac{\partial^{\mu} \varphi \partial^{\nu} \varphi}{\partial_{\alpha} \varphi \partial^{\alpha} \varphi}
$$


Eq. (4.20) contains contributions from both metric and dilaton perturbations, as defined in the longitudinal gauge. In the covariant approach that we are considering, each one of the two contributions cannot be separately computed as it would turn out to be too large to be consistent with a linearized treatment. Only the appropriate combination corresponding to $\Delta$ remains small enough to be treated perturbatively.

Moreover, the spectral distribution (4.19) is the same as the one obtained for $\psi$ and $\chi$ separately, if the growing solution of the perturbation equations is simply neglected [11]. It also corresponds to the dilaton and graviton spectrum obtained via a Bogoliubov transformation, connecting the initial vacuum to the final vacuum state of a radiation-dominated background (i.e. to the spectrum defined with respect to the asymptotic particle content of the amplified fluctuations [11]). This coincidence suggests that the growing mode of scalar metric perturbations does not have any direct physical meaning. If so it should be possible to get rid of it through a suitable coordinate choice. This possibility will be discussed in the next section.

As far as the fluctuations in the "geometric" (scalar curvature) part of the energy density are concerned, the spectral amplitude obtained from $C_{k}$,

$$
\frac{1}{k^{5 / 2}}\left|C_{k}\right| \simeq \frac{H_{1}}{M_{p}}\left|k \eta_{1}\right|^{3 / 2} \ln |k \eta|
$$

exactly reproduces (4.19) (even in the logarithm!) and also coincides with the spectral behaviour of tensor perturbations (see eq. (2.15)). In the case of the $C_{k}$ spectrum we are also not dealing with a purely gravitational energy distribution: again the contributions of metric and dilaton fluctuations, as defined in the longitudinal gauge, are both present and mixed.

We note, finally, that in the linear approximation the fluctuations in both energy density and curvature are defined in terms of the canonical variable $v$. Indeed, from eq. (4.16), (4.19) and the exact definition (4.2), we find

$$
\begin{aligned}
\frac{\delta \rho}{\rho} & \simeq \frac{v}{a} \\
\delta^{(3)} R & \simeq \nabla^{2} \frac{v}{a}
\end{aligned}
$$


The fact that $\Delta$ and $C$ are small may thus be seen to follow from the fact that the contributions of $\psi$ and $\chi$ combine just to give $v$. It is striking that the linearized asymptotic behaviour (4.12) of $\Delta$ and $C$ is correctly given by extrapolating the logarithmic behaviour of $v$, eq. (3.15), to times at which the definition of $v$ in terms of $\psi$ and $\chi$ is no longer consistent with the linear perturbation theory. This suggests that $v$, first identified in [36 as the correct variable for the canonical quantization of perturbations in the linear approximation, could also be an appropriate variable for a consistent perturbative expansion describing the evolution of inhomogeneities in a general scalar-tensor background.

\section{Gauging down the growing mode}

The results of the previous Section strongly suggest that, owing to the special properties of the growing mode, physically observable inhomogeneities stay small at all times. If so, a suitable gauge reflecting that fact should exist. Stated differently, there should be a good coordinate system in which the growing mode of scalar perturbations should be strongly suppressed and metric perturbations can be treated perturbatively throughout their evolution. In this Section we will present a suitable candidate for such a job, the "off-diagonal" gauge. As it turns out, this choice "gauges away" completely the growing mode at $k=0$ and "gauges down" the small- $k$ growing modes. Although this will be shown to be sufficient in order to construct a perturbative solution around a "shifted" background, we will argue that the construction of a systematic expansion in a small parameter may require a further change of coordinates.

We start our search for the desired gauge from the longitudinal gauge (3.4), in which the line element takes the form

$$
d s^{2}=a^{2}(\eta)\left[(1+2 \phi) d \eta^{2}-(1-2 \psi) \delta_{i j} d x^{i} d x^{j}\right]
$$

Consider now the coordinate transformation

$$
\eta \rightarrow \tilde{\eta}=\eta+\theta\left(\eta, x^{i}\right), \tilde{x}^{i}=x^{i}
$$


It is easy to check that, at first order in $\theta, \phi, \psi$, the choice

$$
\theta=-\mathcal{H}^{-1} \psi
$$

brings the line element to the "off-diagonal" form

$$
d s^{2}=a^{2}(\tilde{\eta})\left[\left(1-2 \theta^{\prime}-4 \mathcal{H} \theta+2(\phi-\psi)\right) d \tilde{\eta}^{2}-2 \partial_{i} \theta d x^{i} d \tilde{\eta}-\delta_{i j} d x^{i} d x^{i}\right]
$$

Furthermore, at first order, the relation $\phi=\psi$ holds true for the general solution while, for the growing mode solution, one also finds $\psi^{\prime}=-4 \mathcal{H} \psi$. Using (5.3), this implies $\theta^{\prime}=-2 \mathcal{H} \theta$. Therefore, as far as the growing mode is concerned, the line element (5.4) simply becomes

$$
d s^{2}=a^{2}(\tilde{\eta})\left[\left(1+\mathcal{O}\left(\tilde{\eta}^{2} \partial^{2} \phi\right)\right) d \tilde{\eta}^{2}-\left(\delta_{i j}+\mathcal{O}\left(\tilde{\eta}^{2} \partial_{i} \partial_{j} \phi\right) d x^{i} d x^{i}-2 \partial_{i} \theta d x^{i} d \tilde{\eta}\right]\right.
$$

We see that the dangerously large entries in $\delta g_{00}$ and $\delta g_{i i}$ have been tamed and have given rise to the off-diagonal entry $\partial_{i} \theta=-\mathcal{H}^{-1} \partial_{i} \psi$. For long wavelengths the typical size of the off-diagonal entry, $\delta g_{0 i} \sim|k \eta| \psi$, is a factor $|k \eta|<<1$ smaller than the original perturbation. Even smaller terms appear in $\delta g_{00}, \delta g_{i i}$ and are such that the growing mode is completely gauged-away for an exactly homogeneous perturbation.

The previous result suggests starting the analysis of scalar perturbations directly in a new "off-diagonal" gauge defined, according to the general line element (3.3), by

$$
d s^{2}=a^{2}(\eta)\left[(1+2 \phi) d \eta^{2}-\delta_{i j} d x^{i} d x^{i}-2 \partial_{i} B d x^{i} d \eta\right]
$$

from which the metric perturbations can be read

$$
\begin{aligned}
\delta g_{00} & =2 a^{2} \phi \\
\delta g_{i 0} & =-a^{2} \partial_{i} B \\
\delta g_{i j} & =0
\end{aligned}
$$

and, to first order,

$$
\begin{aligned}
\delta g^{00} & =-2 \phi / a^{2} \\
\delta g^{i 0} & =-\partial_{i} B / a^{2} \\
\delta g^{i j} & =0 .
\end{aligned}
$$


This gauge choice is interesting in itself. It represents a complete gauge choice, namely, it does not contain any residual degrees of freedom and it is similar, in that respect, to the longitudinal gauge. Indeed, under an infinitesimal coordinate transformation which preserve the scalar nature of the perturbations, $x^{\mu} \rightarrow \tilde{x}^{\mu}=x^{\mu}+\epsilon^{\mu}\left(x^{\alpha}\right)$, with $\epsilon^{0}=\epsilon^{0}(\eta, \vec{x})$, $\epsilon^{i}=\partial^{i} \epsilon(\eta, \vec{x})$, the various entries of the general perturbed metric (3.3) transform as follows [6]

$$
\begin{aligned}
\phi \rightarrow \widetilde{\phi} & =\phi-\frac{a^{\prime}}{a} \epsilon^{0}-\epsilon^{0^{\prime}} \\
\psi \rightarrow \widetilde{\psi} & =\psi+\frac{a^{\prime}}{a} \epsilon^{0} \\
E \rightarrow \widetilde{E} & =E-\epsilon \\
B \rightarrow \widetilde{B} & =B+\epsilon^{0}-\epsilon^{\prime}
\end{aligned}
$$

so that the choice $\tilde{E}=0=\tilde{\psi}$ indeed determines the vector $\epsilon^{\mu}$ uniquely.

By perturbing, in this gauge, the Einstein equations around the background (3.2), we obtain from the $(0,0)$ and $(i, i)$ components of eq.(2.1), respectively, the perturbation equations

$$
\begin{gathered}
-4 \mathcal{H} \nabla^{2} B=\chi^{\prime} \varphi^{\prime} \\
4 \mathcal{H} \phi^{\prime}=\chi^{\prime} \varphi^{\prime},
\end{gathered}
$$

from which the simple relation $\phi^{\prime}=-\nabla^{2} B$ also follows. From the $(i, 0)$ and $(i, j \neq i)$ components we obtain, respectively, two constraints expressing $\chi$ and $\phi$ in terms of $B$,

$$
\begin{gathered}
4 \mathcal{H} \phi=\chi \varphi^{\prime} \\
\phi=-\left(B^{\prime}+2 \mathcal{H} B\right)
\end{gathered}
$$

From equations (5.10)-(5.13) it is possible to obtain the following decoupled equation for the Fourier mode $B_{k}$,

$$
B_{k}^{\prime \prime}+2 \mathcal{H} B_{k}^{\prime}+\left(k^{2}-4 \mathcal{H}^{2}\right) B_{k}=0
$$

which, asymptotically $(|k \eta|<<1)$, has the solution

$$
B_{k}=c_{1}(k) \eta \ln |k \eta|+c_{2}(k) \eta^{-1}
$$


One can check that $-\mathcal{H} B=-B / 2 \eta$ has the same asymptotic behaviour as $\psi$ in the longitudinal gauge, i.e., $\psi \sim \eta^{-2}$. This has to be the case since, in the off-diagonal gauge, $-\mathcal{H} B$ corresponds exactly to the Bardeen variable $\Psi$ which, instead, coincides with $\psi$ in the longitudinal gauge [6]. Indeed, adding momentarily a tilde to quantities in the off-diagonal gauge, and using the asymptotic solutions (5.15) and (3.11) (with an obvious rescaling of the integration constants)

$$
\tilde{\Psi}=\tilde{\psi}-\frac{a^{\prime}}{a} \tilde{B}=c_{1} \ln |k \eta|+\frac{c_{2}}{\eta^{2}}=\psi=\Psi
$$

On the other hand, in the off-diagonal gauge (5.7), the growing mode solution does not contribute to the $\delta g_{00}$ and $\delta \varphi$ perturbations. Inserting the solution (5.15) into eqs.(5.12), (5.13) we find the harmless asymptotic behaviour $\phi \simeq \chi \simeq$ const $\times \ln |k \eta|$.

Another interesting property of the off-diagonal gauge is that the canonical variable $v$, instead of having the complicated form of eq.(3.12), is just given by $a \chi$. As a consequence, $\chi$, like $h$ in eq. (1.2), obeys the simple, decoupled equation

$$
\chi^{\prime \prime}+2 \mathcal{H} \chi^{\prime}-\nabla^{2} \chi=0 .
$$

whose asymptotic solution has already been given in eq. (1.3).

Because of the extra power of $|k \eta|$ in the Fourier transform of $\delta g_{i 0}$, the off-diagonal gauge stands a better chance of providing a setup for a reliable linear description of amplified vacuum fluctuations -both for the metric and for the dilaton- when they are far outside the horizon. In order to verify this, we normalize the mode $B_{k}$ to the initial vacuum state by using the asymptotic relation $B=-2 \eta \psi$ (Cf. eq.(5.3)). After inserting the correct normalization of $\psi_{k}$, we obtain, for the typical amplitude of the fluctuations associated with $\nabla B$, over length scales $k^{-1}$,

$$
\left|\delta_{B_{k}}(\eta)\right|=k^{3 / 2}\left|k B_{k}\right| \simeq \frac{H_{1}}{M_{p}}\left|k \eta_{1}\right|^{1 / 2}\left|\frac{\eta_{1}}{\eta}\right|,
$$

which for any $k$ is smaller than 1 for all $|\eta|>\eta_{1}$, namely for the whole duration of the inflationary epoch. An even smaller expression is easily obtained for $\left|\delta_{\phi_{k}}(\eta)\right|$,

$$
\left|\delta_{\phi_{k}}(\eta)\right| \simeq\left(\frac{H_{1}}{M_{p}}\right)\left(k \eta_{1}\right)^{3 / 2} \ln |k \eta| .
$$


A second significant check that amplified vacuum fluctuations do not perturb the homogeneous background in any substantial way follows from an explicit computation of the invariant

$$
W=\frac{\left|C_{\mu \nu \alpha \beta} C^{\mu \nu \alpha \beta}\right|}{\left|R_{\mu \nu} R^{\mu \nu}\right|}
$$

where $C_{\mu \nu \alpha \beta}$ and $R_{\mu \nu}$ are, respectively, the Weyl and Ricci tensors. The unperturbed background is conformally flat and has a vanishing Weyl tensor. Consequently, the invariant $W$ vanishes to zeroth order and to first order in metric perturbations. To second order in metric perturbations, a straightforward but rather long calculation (which we shall not reproduce here) shows that an upper bound on the magnitude of $W$ is given by $W<\left|\partial_{i} B\right|^{2}$. The magnitude of $W$ is thus bounded by the fluctuation $\left|\delta_{B}\right|^{2}$, which, according to eq.(5.18), remains smaller than unity on all scales and at all times $|\eta|>\eta_{1}$. This result represents an additional covariant and gauge-invariant confirmation that the physical manifold can be consistently described, to leading order, in terms of some small inhomogeneity perturbations lying on top of a homogeneous background solution of the string cosmology equations.

A complete check of the validity of the linear approximation of cosmological perturbation expansion requires a full understanding of the formal structure of cosmological perturbation theory beyond leading order, which, unfortunately, is lacking to this date. An important step in this direction, which, to the best of our knowledge, has not been attempted before in any other approach, would consist of a direct comparison between second and first order terms in the perturbed Einstein equations. We have undertaken part of such calculation by computing all quadratic terms in the four independent scalar perturbation equations. The outcome will be discussed below, not before warning the reader that a full second order computation of scalar perturbations should require a generalization of the off-diagonal gauge ansatz for the metric, as well as consideration of the mixing of scalar, tensor and possibly vector perturbations at second order.

We consider, for computational convenience, the following equivalent form of Einstein's equations (2.1),

$$
R_{\mu \nu}-\frac{1}{2} \partial_{\mu} \varphi \partial_{\nu} \varphi=0
$$

whose left hand side we denote, for simplicity, by $E_{\mu \nu}$. For completeness we first write down 
the first order expressions for $E_{\mu \nu}$ which we denote by $E_{\mu \nu}^{(1)}$

$$
\begin{aligned}
& E_{00}^{(1)}=\nabla^{2}\left(B^{\prime}+\mathcal{H} B+\phi\right)+3 \mathcal{H} \phi^{\prime}-\varphi^{\prime} \chi^{\prime} \\
& E_{0 i}^{(1)}=2 \mathcal{H} \phi_{i}-\frac{1}{2} \varphi^{\prime} \chi_{i} \\
& E_{i j}^{(1)}=-\left(B_{i j}^{\prime}+2 \mathcal{H} B_{i j}+\phi_{i j}\right)-\mathcal{H} \delta_{i j}\left(\phi^{\prime}+\nabla^{2} B\right),
\end{aligned}
$$

where subscripts on $B, \phi$ and $\chi$ denote spatial gradients. The solution of the first order equations has already been given in equations (5.12), (5.13) and (5.15). Straightforward but lengthy calculations lead to the following quadratic expressions for the four independent components of $E_{\mu \nu}$, which we denote by $\tilde{E}_{\mu \nu}^{(2)}$ (the full second order $E_{\mu \nu}^{(2)}$ includes, of course, terms linear in the second order corrections to the fluctuations)

$$
\begin{aligned}
E_{00}^{(2)} & =3 \mathcal{H} B_{k}^{\prime} B^{k}-\phi^{\prime} \nabla^{2} B-\phi_{i}^{2}+\mathcal{H} \phi_{k} B^{k}-6 \mathcal{H} \phi \phi^{\prime}-\frac{1}{2}\left(\chi^{\prime}\right)^{2} \\
E_{0 i}^{(2)} & =-\mathcal{H} B_{i}\left(\nabla^{2} B+\phi^{\prime}\right)-B_{k} B_{i k}^{\prime}-B^{k} \phi_{i k}-\phi_{i} \nabla^{2} B- \\
& -4 \mathcal{H} \phi \phi_{i}+\phi^{k} B_{i k}-\frac{1}{2} \chi^{\prime} \chi_{i} \\
E_{i j}^{(2)} & =\left(\phi^{\prime}+\nabla^{2} B\right) B_{i j}-\mathcal{H} \delta_{i j} B^{k} B_{k}^{\prime}-B_{i}^{k} B_{j k}+2 \phi B_{i j}^{\prime}+ \\
& +\mathcal{H} \delta_{i j}\left(\phi_{k} B^{k}+4 \phi \phi^{\prime}+2 \phi \nabla^{2} B\right)+\phi_{i} \phi_{j}+2 \phi \phi_{i j}+4 \mathcal{H} \phi B_{i j}-\frac{1}{2} \chi_{i} \chi_{j}
\end{aligned}
$$

We could now write down the perturbation equations up to second order and try to solve them explicitly. Since, for the time being, we are only interested in an order of magnitude estimate of the second order corrections to the first order solution, we will rather only keep track of anomalously large terms showing that, after an additional coordinate transformation, they all vanish eventually.

Large terms only originate from the growing mode in $B$. Keeping track of those and using the first order equations to simplify the second order equations, we arrive at the following structure for the second order corrections to the metric fluctuations

$$
\begin{aligned}
\delta g_{00} & =2 a^{2}\left[\phi-\frac{1}{2} B_{i} B_{i}+\mathcal{O}\left(\phi^{2}\right)\right] \\
\delta g_{i 0} & =-a^{2}\left[\partial_{i} B+\mathcal{O}\left(\phi B_{i}\right)\right] \\
\delta g_{i j} & =a^{2} \mathcal{O}\left(\delta_{i j} \phi^{2}\right)
\end{aligned}
$$


It is quite remarkable that all the large terms in (5.23) can be cancelled by the single large term $-\frac{1}{2} B_{i} B_{i}$ appearing in $\delta g_{00}$. At first sight this appears to indicate that the perturbative expansion is breaking down, since $B_{i} B_{i}$, when evaluated using the growing mode of $B$, is much larger than $\phi$. Consider, however, the coordinate transformation

$$
\eta \rightarrow \tilde{\eta}=\eta, \quad x^{i} \rightarrow \tilde{x}^{i}=x^{i}+\int^{\eta} B_{i}\left(\eta^{\prime}\right) d \eta^{\prime}
$$

As easily verified, this transformation eliminates simultaneously both the contribution of the growing mode of $B$ to the off-diagonal $\left(d \eta, d x^{i}\right)$ entry and the nasty $-\frac{1}{2} B_{i} B_{i}$ term in $\delta g_{00}$, leaving only small corrections to the linear $\phi$ term as well as other small non-diagonal terms, typically containing two spatial derivatives acting on $B$. This cancellation is highly non-trivial and depends crucially on the value $-\frac{1}{2}$ of the coefficient of $B_{i} B^{i}$ at second order in $\delta g_{00}$ (see eq.(5.24)). After performing the coordinate transformation (5.25), the new metric perturbations (indicated by a tilde) up to second order have the following structure

$$
\begin{aligned}
\delta g_{00} & =2 a^{2}\left[\phi+\mathcal{O}\left(\phi^{2}\right)\right] \\
\delta g_{i 0} & =-a^{2}\left[\mathcal{O}\left(\phi B_{i}\right)\right] \\
\delta g_{i j} & =2 a^{2}\left[\int^{\eta} B_{i j} d \eta^{\prime}-\frac{1}{2} \int^{\eta} B_{i k}\left(\eta^{\prime}\right) d \eta^{\prime} \int^{\eta} B_{j k}\left(\eta^{\prime}\right) d \eta^{\prime}+\mathcal{O}\left(\delta_{i j} \phi^{2}\right)\right]
\end{aligned}
$$

We conclude that, after suitable coordinate transformations, all second-order corrections are down by at least an extra factor $\phi$ ( or $\int d \eta B_{i j} \sim \phi$ ) relative to first order, i.e. are genuinely small.

The above second order calculation provides additional support to the conclusion that a linearized description of scalar perturbations is generically adequate in an appropriate gauge. Of course, the calculation should not be interpreted as suggesting that perturbation theory will be uniformly good at all scales. At second order different modes (as well as different angular momenta) couple in a non-trivial way and it is not excluded that regions of the spectrum which are very depressed at first order will get larger contributions from second order corrections. Investigating whether this phenomenon could enhance the power spectrum at very small $k$ is left as an interesting subject for future research. 


\section{Summary and conclusions}

As mentioned already in the introduction, the cosmological equations obtained from the low energy string effective action imply that an arbitrarily small, finite density of string matter is enough to trigger the evolution of the perturbative string vacuum (taken as initial state) towards a regime of growing string coupling and curvature. Such a regime eventually evolves into a long period of dilaton-driven inflation [11, 13]. This final epoch corresponds to a phase of accelerated expansion in the S-frame (of accelerated contraction in the E-frame) and is invariantly characterized by shrinking event horizons [9]-[11], in contrast with the constant event horizon of the more conventional de Sitter-like inflation. In such a context, the external metric and dilaton background fields contribute jointly to the parametric amplification of metric perturbations [23].

In this paper we have considered and contrasted tensor and scalar metric perturbations showing that, while for the former a straightforward computation is possible, for the latter some special care is needed owing to the presence of rapidly growing modes in the most conventional parametrization of the metric fluctuations (longitudinal gauge). Nonetheless, we have been able to compute the scalar perturbation spectrum using either an appropriate set of covariant and gauge invariant variables [29], or by using a new gauge, which we found to be particularly useful for the purpose of keeping scalar metric perturbations small.

In spite of their very different treatment, tensor and scalar perturbations are predicted to have very similar amplitudes and spectra, given by (see eqs. (2.15) and (4.19)):

$$
\left|\delta_{h_{k}}(\eta)\right| \simeq\left(\frac{H_{1}}{M_{p}}\right)\left(k \eta_{1}\right)^{3 / 2} \ln |k \eta|
$$

and

$$
k^{3 / 2}\left|\frac{\delta \rho_{k}}{\rho}\right| \simeq \frac{1}{\sqrt{k}}\left|\Delta_{k}\right| \simeq \frac{H_{1}}{M_{p}}\left|k \eta_{1}\right|^{3 / 2} \ln |k \eta|
$$

These perturbations should manifest themselves in (at least) two different ways: on one hand, as metric perturbations at the surface of last scattering, they will affect the homogeneity of the CMB spectrum (through the Sachs-Wolfe effect). As discussed elsewhere [9], such an effect will be small at the scales measured by COBE, even if one takes $H_{1} \sim$ 
$M_{p}$. This is simply because our spectrum, unlike the one of (almost) de-Sitter inflation, is decreasing with the length-scale of the perturbation.

The second effect is a background of relic gravitons and dilatons which should be still around us having been left over from the "Planck-String" era. Quite amusingly, both spectra bear a strong resemblance to the (unperturbed) Planckian spectrum of the CMB photons themselves. Indeed, the above equations readily lead to the graviton-dilaton spectral-energy distribution:

$$
\frac{d \rho}{d \ln \omega} \simeq \omega^{4}\left(\frac{\omega_{1}}{\omega}\right) \ln ^{2}\left(\frac{\omega_{1}}{\omega}\right),
$$

at $\omega<\omega_{1}$ (and exponentially suppressed at $\omega>\omega_{1}$ ). Here $\omega_{1}$ is (the present value of) the maximal amplified (red-shifted) proper frequency. Assuming the end of dilaton-driven inflation to be quickly followed by the standard radiation-dominated era (which certainly needs not be the case), one finds $\omega_{1} \equiv a_{1} H_{1} / a \simeq 10^{11}\left(H_{1} / M_{p}\right)^{1 / 2} H e r t z$. Eq. (6.3) can be compared to the CMB spectrum:

$$
\frac{d \rho_{c m b}}{d \ln \omega} \simeq \omega^{4}\left(e^{\frac{\omega}{T_{\gamma}}}-1\right)^{-1} .
$$

Modulo logarithms, the two spectra agree with each other but, of course, there is no reason to expect $T_{\gamma} \simeq 2.7 \mathrm{~K}$ to be very close to $\omega_{1}$ since gravitons decoupled very early from everything else while photons underwent a complicated history until decoupling. Yet, amusingly enough, if $H_{1} \sim M_{p}$, the expected value of $T_{g r} \equiv \omega_{1}$ (under the assumption of a quick transition to radiation dominance) is of the same order as $T_{\gamma}$.

Like any Planckian spectrum, our graviton-dilaton spectrum is also strongly tilted towards large wavenumbers with a spectral index $n=4$, in contrast to the de-Sitter case $(n=1)$ and in agreement with previous computations on the rate of graviton and dilaton production in a string cosmology context [11, 37]. These tilted spectra contain most of their total power near the maximal proper frequency $\omega_{1}$. It would be an interesting challenge to conceive experimental apparatuses able to detect a relic stochastic gravitational background of such an intensity and in such a high-frequency range. 


\section{Appendix}

\section{Scalar perturbations in higher-dimensional back- grounds}

We shall first discuss the growth of scalar perturbations, in the longitudinal gauge, for isotropic, dilaton-driven backgrounds with $d>3$ spatial dimensions. In $d$ dimensions eq. (3.9) is modified: by combining the $d$-dimensional generalization of the perturbation equations (3.5)-(3.8) and of the background equations (3.2) we get for $\psi_{k}$ (see for instance [11])

$$
\psi_{k}^{\prime \prime}+3(d-1) \mathcal{H} \psi_{k}^{\prime}+k^{2} \psi_{k}=0
$$

which, for $|k \eta|<<1$, has the asymptotic solution

$$
\psi_{k}=c_{1}+c_{2} \frac{\eta}{a^{3(d-1)}}
$$

In $d$-dimensions also the inflationary background solution is modified [1]

$$
a=(-\eta)^{1 /(d-1)}, \quad \varphi=-\sqrt{2 d(d-1)} \ln a
$$

As a consequence, the scalar mode is still growing asymptotically as $\eta^{-2}$, exactly as in $d=3$. The normalized spectral amplitude acquires however a $d$-dependence, since the expression of $\psi$ in terms of the variable $v$ satisfying canonical commutation relations becomes 38]

$$
\psi_{k}=\frac{1}{2(d-1) k^{2}} \frac{\varphi^{\prime 2}}{\mathcal{H}}\left(\frac{v_{k}}{z}\right)^{\prime}, \quad z=\frac{\varphi^{\prime}}{\mathcal{H}} a^{(d-1) / 2}
$$

where

$$
v=a^{(d-1) / 2}\left(\chi+\frac{\varphi^{\prime}}{\mathcal{H}}\right), \quad v_{k}^{\prime \prime}+\left(k^{2}-\frac{z^{\prime \prime}}{z}\right) v_{k}=0, \quad \frac{z^{\prime \prime}}{z}=\frac{(d-1)^{2}}{4(d-2)} \frac{a^{\prime \prime}}{a}
$$

The solution for $v_{k}$ in the small $|k \eta|$ limit,

$$
v_{k}=c_{1} z+c_{2} z \int^{\eta} \frac{d \eta^{\prime}}{z^{2}\left(\eta^{\prime}\right)}
$$

corresponds then to the normalized asymptotic behaviour

$$
\left|v_{k}\right|=\frac{z}{z_{\mathrm{HC}}} \frac{|\ln (-k \eta)|}{\sqrt{k}}
$$


which inserted into eq. (A.4) leads to the typical fluctuation amplitude, on scales $k^{-1}$,

$$
\left|\delta_{\psi_{k}}(\eta)\right| \simeq\left(\frac{H_{1}}{M_{p}}\right)^{(d-1) / 4} \frac{\left(k \eta_{1}\right)^{d / 2}}{(k \eta)^{2}}
$$

The condition $\left|\delta_{\psi}\right| \lesssim 1$ implies

$$
\left|\frac{\eta}{\eta_{1}}\right| \gtrsim\left(\frac{H_{1}}{M_{p}}\right)^{(d-1) / 4}\left(\frac{k}{k_{1}}\right)^{(d-4) / 4}
$$

which is satisfied, if the inflationary evolution is switched off at a scale $H_{1} \sim\left(a_{1} \eta_{1}\right)^{-1} \leq M_{P}$, for all $d \geq 4$. Though in a higher dimensional background the presence of the growing mode is not eliminated, the use of the linearized metric perturbation approach is nevertheless allowed.

The growing mode, which has been shown to be present with the same time-dependence for any number of spatial dimensions (see eq. (A.1), cannot be eliminated even by relaxing the isotropy assumption. In order to discuss this point we shall consider scalar perturbations in the anisotropic background (2.3), (2.5), by setting (in the longitudinal gauge),

$$
\delta \varphi=\chi, \quad \delta g_{00}=2 a^{2} \phi, \quad \delta g_{i j}=2 a^{2} \psi \delta_{i j}, \quad \delta g_{m n}=2 b^{2} \xi \delta_{m n}
$$

with $\delta g_{0 m}=0=\delta g_{i m}$. This choice is certainly justified if, in a dimensional reduction context, we consider perturbations which are only function of time and of the external coordinates $x^{i}, i=1, \ldots, d$. The $(i, j \neq i)$ component of the perturbed Einstein equations gives then a relation between the three perturbation variables

$$
\phi=(d-2) \psi+n \xi
$$

which allows us to eliminate $\phi$ everywhere in the perturbation equations. The $(0, i)$ components give the constraint

$$
\begin{aligned}
(d-1) \psi^{\prime}+(d-2) \psi[(d-1) \mathcal{H}+n \mathcal{F}] & + \\
+n \xi^{\prime}+n \xi[(d-2) \mathcal{H}+(n+1) \mathcal{F}] & =\frac{1}{2} \varphi^{\prime} \chi
\end{aligned}
$$

and the $(0,0)$ component gives

$$
\begin{aligned}
(d-1) \nabla^{2} \psi-\psi^{\prime}[d(d-1) \mathcal{H}+n \mathcal{F}] & + \\
+n \nabla^{2} \xi-n \xi^{\prime}[d \mathcal{H}+(n-1) \mathcal{F}] & =\frac{1}{2} \varphi^{\prime} \chi^{\prime}
\end{aligned}
$$


The perturbation of the dilaton equation (2.2) gives

$$
\chi^{\prime \prime}+[(d-1) \mathcal{H}+n \mathcal{F}] \chi^{\prime}-\nabla^{2} \chi=2 \varphi^{\prime}\left[(d-1) \psi^{\prime}+n \xi^{\prime}\right]
$$

Finally, the $(i, i)$ and $(m, m)$ components of the perturbed Einstein equations, combined with eq. (A.13), provide the following interesting system of coupled equations for the "external" and "internal" perturbations $\psi$ and $\xi$ [38]:

$$
\begin{aligned}
& (d-1)\left\{\square \psi+\psi^{\prime}[3(d-1) \mathcal{H}+3 n \mathcal{F}]\right\}= \\
& =-n\left\{\square \xi+\xi^{\prime}[3(d-1) \mathcal{H}+3 n \mathcal{F}]\right\} ; \\
& d\left\{\square \psi+\psi^{\prime}\left[3(d-1) \mathcal{H}+\frac{\mathcal{F}}{d}(2(d-1)(n-1)+n d)\right]\right\}= \\
& =-(n-1)\left\{\square \xi+\xi^{\prime}\left[\frac{\mathcal{H}}{n-1}(3 d n-d-n+1)+3 n \mathcal{F}\right]\right\}
\end{aligned}
$$

where $\square \equiv \frac{\partial^{2}}{\partial \eta^{2}}-\nabla^{2}$.

This system can be easily diagonalized to find the (time-dependent) linear combination of $\psi$ and $\xi$ representing the true "propagation eigenstates". For our purpose, however, the asymptotic behaviour of the modes $\psi_{k}, \xi_{k}$ can be simply obtained by inserting into the previous system the ansatz

$$
\psi_{k}=A(-\eta)^{x}, \quad \xi_{k}=B(-\eta)^{x}
$$

One then finds from eqs. (A.15) that in the $|k \eta| \rightarrow 0$ limit there are non trivial solutions for the coefficients $A$ and $B$ only if $x=0$ or $x=-2$, which means that, asymptotically,

$$
\psi_{k}=A_{1}+\frac{A_{2}}{\eta^{2}}, \quad \xi_{k}=B_{1}+\frac{B_{2}}{\eta^{2}}
$$

We thus find for the scalar perturbation modes the same asymptotic growth, with the same power-like behaviour in $\eta$, as in the previous case of $d=3$ isotropic dimensions. 


\section{References}

[1] L. P. Grishchuk, Sov. Phys. JEPT 40, 409 (1975);

A.A. Starobinski, JEPT. Lett. 30, 682 (1979).

[2] A. Guth, Phys. Rev. D23, 347 (1981);

A. Linde, Phys. Lett 108B, 389 (1982);

A. Albrecht and P.J. Steinhardt, Phys. Rev. Lett. 48, 122 (1982);

A. Linde, Phys. Lett 129B, 177 (1983).

[3] V. Mukhanov and G. V. Chibisov, JETP Lett. 33, 532 (1981);

A. Guth and S. Y. Pi. Phys. Rev. Lett. 49, 1110 (1982);

A.A. Starobinski, Phys. Lett. B117, 175 (1982);

S.W. Hawking, Phys. Lett. B115, 295 (1982);

J.M. Bardeen, P.S. Steinhardt and M.S. Turner, Phys. Rev. D28, 679 (1983).

[4] A. Linde, Phys. Lett. B116, 335 (1982);

G.V. Chibisov and V.N. Mukhanov, Mon. Not. R. Astron. Soc. 200, 535 (1982);

A.A. Starobinski, Pisma Astron. Zh. 9, 579 (1983);

R. Brandenberger, Nucl. Phys. B245, 328 (1984);

D.H. Lyth, Phys. Rev. D31, 1792 (1985);

M. Sasaki, Prog. Theor. Phys. 76, 1036 (1986).

[5] V. A. Rubakov, M. Sazhin and A. Veryaskin, Phys. Lett. B115, 189 (1982);

R. Fabbri and M. Pollock, Phys. Lett. B 125, 445 (1983);

L. Abbott and M. Wise, Nucl. Phys. B244, 541 (1984);

L. P. Grishchuk, Sov. Phys. Usp. 31, 940 (1988);

L. P. Grishchuk and M. Solokin, Phys. Rev. D43, 2556 (1991).

[6] V. Mukhanov, H.A. Feldman and R. Brandenberger, Phys. Rep. 215, 203 (1992).

[7] M. Gasperini, N. Sanchez and G. Veneziano, Nucl. Phys. B364, 365 (1991).

[8] G. Veneziano, Phys. Lett. B265, 287 (1991). 
[9] M. Gasperini and G. Veneziano, Astropart. Phys. 1, 317 (1993).

[10] M. Gasperini and G. Veneziano, Mod. Phys. Lett. A8, 3701 (1993).

[11] M. Gasperini and G. Veneziano, Phys. Rev. D50, 2519 (1994)

[12] R. Brustein and G. Veneziano, Phys. Lett. B329, 429 (1994).

[13] M. Gasperini, "Phenomenological aspects of the pre-big-bang scenario in string cosmology", in Proc. of the 2nd Journée Cosmologie, Paris, June 1994 (World Scientific P.C., Singapore), Torino University Preprint DFTT-24/94, (astro-ph/9406056);

M. Gasperini, "The inflationary role of the dilaton in string cosmology", in Proc. of the First Int. Workshop on Birth of the Universe and Fundamental Physics, Rome, May 1994 (Springer-Verlag, Berlin), Torino University Preprint DFTT-29/94, astro$\mathrm{ph} / 9407026)$.

[14] G. Veneziano, "Strings, Cosmology, ...and a Particle" talk given at PASCOS '94, preprint CERN-TH.7502/94 (November 1994).

[15] R. Brustein, "The role of the superstring dilaton in cosmology and particle physics", in Proc. of the XXIX Rencontres de Moriond, Meribel, March 1994;

R. Brustein, "Cosmology and models of supersymmetry breaking in string theory", Proc. of the SUSY94 Workshop, Ann Arbor, May 1994.

[16] K. Behrndt and S. Forste, Nucl. Phys. B430 (1994) 441;

E. J. Copeland, A. Lahiri and D. Wands, Phys.Rev.D50 (1994) 4880;

C. Angelantonj, L. Amendola, M. Litterio and F. Occhionero, "String cosmology and inflation", FERMILAB-Pub-94/315-A (September 1994).

[17] J. Levin and K. Freese, Nucl. Phys. B421 (1994) 635.

[18] N. Sanchez and G. Veneziano, Nucl. Phys. B333 (1990) 253.

[19] D. Sahdev, Phys. Lett. B137, 155 (1984);

R. B. Abbott, S. M. Barr and S. D. Ellis, Phys. Rev. D30, 720 (1984); 
E. W. Kolb, D. Lindley and D. Seckel, Phys. Rev. D30, 1205 (1984);

F. Lucchin and S. Matarrese, Phys. Lett. B164, 282 (1985).

[20] E. Kiritsis and K. Kounnas, Phys. Lett. B331 (1994) 51;

E. Kiritsis and K. Kounnas, in Proc. of the 2nd Journée Cosmologie, Paris, June 1994 (World Scientific P.C., Singapore), Preprint CERN-TH.7328/94, (hep-th/9407005).

[21] A. A. Tseytlin, Phys. Lett. B334 (1994) 315.

[22] E. Martinec, "Spacelike singularities and string theory" Preprint EFI-94-62, December 1994 (hep-th/9412074).

[23] M. Gasperini and M. Giovannini, Phys. Rev. D47, 1529 (1993).

[24] L. P. Grishchuk and Y. V. Sidorov, Phys. Rev. D42, 3413 (1990);

L. P. Grishchuk, H. A. Haus and K. Bergman, Phys. Rev. D46, 1440 (1992).

[25] J. D. Barrow, J. P. Mimoso and M. R. de Garcia Maia, Phys.Rev. D48, 3630 (1993).

[26] J. Bardeen, Phys. Rev. D22, 1822 (1980);

M. Sasaki, Prog. Theor. Phys. 70, 394 (1983).

[27] R. B. Abbott, B. Bednarz and S. D. Ellis, Phys. Rev. D33, 2147 (1986).

[28] G.F.R. Ellis and M. Bruni, Phys. Rev. D40, 1804 (1989).

[29] M. Bruni, G. F. R. Ellis and P. K. S. Dunsby, Class. Quantum Grav. 9, 921 (1992).

[30] S. W. Hawking, Astrophys. J. 145, 544 (1966).

[31] A. R. Liddle and D. H. Lyth, Phys. Rep. 231, 1 (1993).

[32] C. Lovelace, Phys. Lett. B135, 75 (1984);

E. S. Fradkin and A. A. Tseytlin, Nucl. Phys. B261, 1 (1985);

C. G. Callan, D. Friedan, E. J. Martinec and M. J. Perry, Nucl. Phys. B262, 593 (1985).

[33] M. Muller, Nucl. Phys. B337, 37 (1990). 
[34] K. A. Meissner and G. Veneziano, Mod. Phys. Lett. A6, 3397 (1991).

[35] M. Demianski, A. G. Polnarev and P. Naselski, Phys. Rev. D47, 5275 (1993).

[36] V. F. Mukhanov, Sov. Phys. JEPT 67, 1297 (1988).

[37] M. Gasperini, Phys. Lett. B327, 214 (1994).

[38] M. Giovannini, PhD Thesis (in preparation). 